\title{
EXISTENCE OF A MULTIPLICATIVE BASIS FOR A FINITELY SPACED MODULE OVER AN AGGREGATE
}

\author{
A. V. Roiter and V. V. Sergeichuk
}

UDC 519.1

\begin{abstract}
It is proved that a finitely spaced module over a $k$-category admits a multiplicative basis (such a module gives rise to a matrix problem in which the allowed column transformations are determined by a module structure, the row transformations are arbitrary, and the number of canonical matrices is finite).
\end{abstract}

It was proved in [1] that a finite-dimensional algebra having finitely many isoclasses of indecomposable representations admits a multiplicative basis. In [2, Secs.4.10-4.12], an analogous hypothesis was formulated for finitely spaced modules over an aggregate and an approach to its proof was proposed. The purpose of the present paper is to prove this hypothesis. Below, $k$ always denotes an algebraically closed field.

Let us recall some definitions given in [2] (see also [3]).

By definition, an aggregate $\mathcal{A}$ over $k$ is a category that satisfies the following conditions:

(a) for each $X, Y \in \mathcal{A}$, the set $\mathcal{A}(X, Y)$ is a finite-dimensional vector space over $k$ :

(b) the composition maps are bilinear;

(c) $\mathcal{A}$ has finite direct sums;

(d) each idempotent $e \in \mathcal{A}(X, X)$ has a kernel.

As a consequence, each $X \in \mathcal{A}$ is a finite direct sum of indecomposables and the algebra of endomorphisms of each indecomposable is local.

We denote by $\mathcal{I} \mathcal{A}$ a spectroid of $\mathcal{A}$, i.e., a full subcategory formed by chosen representatives of the isoclasses of indecomposables; let $\mathscr{R}_{\mathcal{A}}$ be the radical of $\mathcal{A}$ Suppose that $\mathcal{I A}$ has finitely many objects. For each $a, b \in \mathcal{I A}$, the space $\mathcal{R}_{\mathfrak{A}}(a, b)$ consists of all uninvertible morphisms of $\mathcal{A}(a, b)$; therefore, $\mathcal{A}(a, b)=\mathcal{R}_{\mathfrak{A}}(a, b)$ for $a \neq b$, $\mathcal{A}(a, a)=k 1_{a} \oplus_{k} \mathcal{R}_{\mathcal{A}}(a, a)$.

A module $M$ over an aggregate $\mathcal{A}$ consists of finite-dimensional vector spaces $M(X)$, one for each object $X \in \mathcal{A}$, and of the linear maps $M(f): M(X) \rightarrow M(Y), m \mapsto f m, f \in \mathcal{A}(X, Y)$, which satisfy the standard axioms, namely,

$$
1_{X} m=m, \quad(f+g) m=f m+g m, \quad(g f) m=g(f m), \quad f(\alpha m)=\alpha(f m)=(\alpha f) m, \quad \alpha \in k .
$$

It gives a $k$-linear functor from $\mathcal{A}$ into the category $\bmod k$ of finite-dimensional vector spaces over $k$. We say that a module $M$ over $\mathcal{A}$ is faithful if $M(f) \neq 0$ for each nonzero $f \in \mathcal{A}(X, Y)$.

Define the basis of $(M, \mathcal{A})$ as a set $\left\{m_{i}^{a}, f_{l}^{b a}\right\}$ consisting of bases $m_{1}^{a}, m_{2}^{a}, \ldots$ of the spaces $M(a)$, $a \in \mathcal{I A}$, and bases $f_{1}^{b a}, f_{2}^{b a}, \ldots$ of the spaces $\mathcal{R}_{\mathcal{A}}(a, b), a, b \in \mathcal{I A}$. The maximal rank of $M\left(f_{l}^{b a}\right)$ is called the rank of a basis. A basis is called a scalarly multiplicative basis if it satisfies the following conditions:

Institute of Mathematics, Ukrainian Academy of Sciences, Kiev. Published in Ukrainskii Matematicheskii Zhumal, Vol. 46, No. 5, pp. 567-579, May, 1994. Original article submitted June 23, 1992. 
(a) each morphism $f_{l}^{b a}$ is thin, i.e., the equality $f_{l}^{b a}=g+h$ implies that $\operatorname{rank} M\left(f_{l}^{b a}\right) \leq \operatorname{rank} M(g)$ and $\operatorname{rank} M\left(f_{l}^{b a}\right) \leq \operatorname{rank} M(h)$ for all $g, h \in \mathcal{A}(a, b)$;

(b) each product $f_{l}^{b a} m_{i}^{a}$ has the form $\lambda m_{p}^{b}, \lambda \in k$;

(c) $f_{l}^{b a} m_{i}^{a}=\lambda m_{p}^{b}, f_{l}^{b a} m_{j}^{a}=\mu m_{p}^{b}$, and $\lambda, \mu \in k \backslash\{0\}$ imply $i=j$.

We say that the basis is multiplicative if each nonzero product $f_{l}^{b a} m_{i}^{a}$ is a basis vector $m_{p}^{b}$.

Denote by $M^{k}$ the aggregate formed by all triples $(V, h, X)$, where $V \in \bmod k, X \in \mathcal{A}$, and $h \in$ $\operatorname{Hom}_{k}(V, M(X))$. A morphism from $(V, h, X)$ to $\left(V^{\prime}, h^{\prime}, X^{\prime}\right)$ is defined by the pair of morphisms $\varphi \in$ $\operatorname{Hom}_{k}\left(V, V^{\prime}\right)$ and $\xi \in \mathcal{A}\left(X, X^{\prime}\right)$ such that $h^{\prime} \varphi=M(\xi) h$. These triples are called spaces on $M$. We say that $M$ is finitely spaced if $M^{k}$ has a finite spectroid.

The present paper deals with the proof of the following theorem:

Theorem. If $M$ is a faithful finitely spaced module over an aggregate $\mathcal{A}$, then $(M, \mathcal{A})$ admits a multiplicative basis of rank $\leq 2$.

We wish to express our deep gratitude to P. Gabriel, Th. Brüstle, T. Guidon, and U.Hassler for discussions and essential corrections.

\section{Construction of a Scalarly Multiplicative Basis}

In Secs. 1-3, $M$ always denotes a finitely spaced module over an aggregate $\mathcal{A}$.

As shown in $[2$, Secs. 4.7,4.8], for each $a \in \mathcal{I A}$, the space $M(a)$ has dimension $d(a) \leq 3$ and a sequence $m_{1}, m_{2}, \ldots, m_{d(a)}$, where

$$
m_{i} \in\left(R_{\mathfrak{A}}(a, a)\right)^{i-1} M(a) \backslash\left(\mathcal{R}_{\mathfrak{A}}(a, a)\right)^{i} M(a),
$$

is a basis in $M(a)$. It is called a triangular basis because the matrix of each map $M(f), f \in \mathcal{A}(a, a)$, has a lower triangular form. We assume that each basis $m_{1}^{a}, \ldots, m_{d(a)}^{a}$ in a scalarly multiplicative basis is triangular (it is always triangular up to permutations of vectors).

A scalarly multiplicative basis is called normed if it satisfies the following condition:

(d) $f_{l}^{b a} m_{i}^{a}=\lambda m_{p}^{b}$ and $\lambda \notin\{0,1\}$ imply that $f_{l}^{b a} m_{i^{\prime}}^{a}=m_{p^{\prime}}^{b}$ for some $i^{\prime}<i$.

A scalarly multiplicative basis can be reduced to a normed basis by means of multiplication of $f_{l}^{b a}$ by scalars. A scalarly multiplicative basis is called reduced if it satisfies condition (d) and the following condition:

(e) If a morphism

$$
\varphi=\sum_{l} \lambda_{l} f_{l}^{b a}
$$

is a product of basis morphisms, then

$$
\operatorname{rank} M(\varphi)=\sum_{\lambda_{l} \neq 0} \operatorname{rank} M\left(f_{l}^{b a}\right)
$$


At the end of this section, we prove that every multiplicative basis of $(M, \mathcal{A})$ is reduced if $\operatorname{char}(k) \neq 2$.

Let $m_{1}^{a}, \ldots, m_{d(a)}^{a}$ be a fixed triangular basis of $M(a)$ for each $a \in \mathcal{I A}$. For $m_{j}^{a}$ and $m_{i}^{b}$, we define a linear map $e_{i j}^{b a}: M(a) \rightarrow M(b)$ such that $e_{i j}^{b a} m_{j}^{a}=m_{i}^{b}$ and $e_{i j}^{b a} m_{j^{\prime}}^{a}=0$ for all $j^{\prime} \neq j$.

Let $f \in R_{\mathcal{A}}(a, b), a, b \in \mathcal{J A}$. We say that $f$ is a short morphism if $f \notin \mathcal{R}_{\mathcal{A}}(c, b) \mathcal{R}_{\mathcal{A}}(a, c)$ for all $c \in \mathcal{A}, f$ is a prime morphism if $M(f)=e_{i j}^{b a}$, and $f$ is a double morphism if

$$
M(f)=e_{i j}^{b a}+\lambda e_{i^{\prime} j^{\prime}}^{b a}, \quad e_{i j}^{b a} \notin M(a, b), \quad i<i^{\prime}, \quad j<j^{\prime}, \quad 0 \neq \lambda \in k .
$$

The coefficient $\lambda$ is called the parameter of a double morphism.

Proposition 1. A set $\left\{m_{i}^{a}, f_{l}^{b a}\right\}$ is a normed (reduced, respectively) scalarly multiplicative basis if and only if the following two conditions are satisfied:

(i) $m_{1}^{a}, m_{2}^{a}, \ldots$ is a triangular basis of $M(a), a \in \mathcal{I A}$;

(ii) $f_{1}^{b a}, f_{2}^{b a}, \ldots$ is the set of all prime and double morphisms of $\mathcal{A}(a, b), a, b \in \mathcal{J A}$, except for one double morphism (one short double morphism, respectively) if the number of double morphisms is equal to three. Moreover, the number of double morphisms of $\mathcal{A}(a, b)$ is equal to zero, one, or three, and, in the last case, there exists a short double morphism.

The statement of Proposition 1 about a normed scalarly multiplicative basis follows from Lemmas 1 and 5 . The complete proof of Proposition 1 will be given in Sec. 2 .

Lemma 1. If $d(a)=2$, then $M(a, a)=k 1_{M(a)}+k e_{21}^{a a}$. If $d(a)=3$, then $M(a, a)=k 1_{M(a)}+k e_{21}^{a a}+$ $k e_{32}^{a a}$ or

$$
M(a, a)=k 1_{M(a)}+k\left(e_{21}^{a a}+\lambda_{a a} e_{32}^{a a}\right)+k e_{31}^{a a}
$$

and $0 \neq \lambda_{a a} \in k$.

The proof of Lemma 1 is obvious.

For every linear map $\varphi: M(a) \rightarrow M(b)$, we denote by $\varphi_{i j} \in k e_{i j}^{b a}$ the linear maps such that $\varphi=\sum \varphi_{i j}$. On $\{1,2, \ldots, d(b)\} \times\{1,2, \ldots, d(a)\}$, we introduce an order relation by $(i, j) \geq(l, r)$ if $i \leq l$ and $j \geq r$. A pair $(l, r)$ is called a step of $\varphi \in M(a, b)$ if $\varphi_{l r} \neq 0$ and $\varphi_{i j}=0$ for all $(i, j)>(l, r)$. A pair $(l, r)$ is called a step of $M(a, b)$ if $\psi_{l r} \neq 0$ for some $\psi \in M(a, b)$ and $\varphi_{i j}=0$ for all $\varphi \in M(a, b)$ and all $(i, j)>(l, r)(l \geq r$ because each basis $m_{1}^{a}, m_{2}^{a}, \ldots$ is triangular).

Lemma 2. If $a, b \in \mathcal{I A}, a \neq b, d(a)=d(b)=3$, and $M(a, b)$ has two steps $(1,2)$ and $(2,3)$, then $M(b, a)=k e_{31}^{a b}$.

Proof. Let $\psi \in M(b, a)$. There exists $\varphi \in M(a, b)$ having the steps $(1,2)$ and $(2,3)$. By Lemma 1 , there exist $\varepsilon \in M(a, a)$ and $\delta \in M(b, b)$ such that $\varphi^{\prime}=\varphi \varepsilon+\delta \varphi$ has the steps $(1,1),(2,2)$, and $(3,3)$. The inclusion $\mathcal{A}(b, a) \mathcal{A}(a, b) \subset \mathcal{R}_{\mathfrak{A}}(a, a)$ implies that

$$
M(b, a) M(a, b) \subset M\left(R_{\mathcal{A}}(a, a)\right)=k e_{21}^{a a} \oplus k e_{31}^{a a} \oplus k e_{32}^{a a} .
$$


Since $\psi \varphi^{\prime} \in M\left(R_{\mathcal{A}}(a, a)\right)$, all steps of $\psi$ are not higher than $(2,1)$ and $(3,2)$. Since $\psi \varphi \in M\left(\mathscr{R}_{\mathfrak{A}}(a, a)\right)$, we have $\psi \in k e_{31}^{a b}$.

Therefore, $M(b, a) \subset k e_{31}^{a b}$. Assume that $M(b, a)=0$. Let us examine the space $\mathcal{H}_{\lambda}=\left(k^{6}, h_{\lambda}, a^{2} \oplus\right.$ $\left.b^{2}\right) \in M^{k}$, where

$$
k^{6}=k \oplus k \oplus k \oplus k \oplus k \oplus k, \quad a^{2}=a \oplus a, \quad b^{2}=b \oplus b, \lambda \in k,
$$

and $h_{\lambda}$ is a linear mapping of $k^{6}$ into

$$
M\left(a^{2} \oplus b^{2}\right)=\left(k m_{1}^{a}\right)^{2} \oplus\left(k m_{2}^{a}\right)^{2} \oplus\left(k m_{3}^{a}\right)^{2} \oplus\left(k m_{1}^{b}\right)^{2} \oplus\left(k m_{2}^{b}\right)^{2} \oplus\left(k m_{3}^{b}\right)^{2}
$$

with the matrix

$$
\left(\begin{array}{l}
1 \\
0
\end{array}\right) \oplus\left(\begin{array}{l}
0 \\
1
\end{array}\right) \oplus\left(\begin{array}{ll|ll}
1 & 0 & 1 & 0 \\
0 & 1 & 0 & 1
\end{array}\right)^{T} \oplus\left(\begin{array}{l}
1 \\
1
\end{array}\right) \oplus\left(\begin{array}{l}
1 \\
\lambda
\end{array}\right) .
$$

We show that $\mathcal{H}_{\lambda} \neq \mathcal{H}_{\mu}$ if $\lambda \neq \mu$. Let $(\varphi, \xi)$ be an isomorphism $\mathcal{H}_{\lambda} \rightarrow \mathcal{H}_{\mu}$. The linear mapping $M(\xi)$ has the block matrix $\left(K_{i j}\right), i, j \leq 6$, where $K_{i j}$ are $2 \times 2$ matrices. By the equality $M(b, a)=0$ and Lemma 1 , we have $K_{i j}=0$, provided that $i<j$. Evidently, $K_{11}=K_{22}=K_{33}, K_{44}=K_{55}=K_{66}$, and $K_{43}=0$.

Since $h_{\mu} \varphi=M(\xi) h_{\lambda}$, the matrix of the nondegenerate mapping $\varphi$ also has the block form $\left(\Phi_{i j}\right), i, j \leq 5$, where the blocks $\Phi_{11}, \Phi_{22}, \Phi_{44}$, and $\Phi_{55}$ are $1 \times 1$ matrices, the block $\Phi_{33}$ is a $2 \times 2$ matrix, and $\Phi_{i j}=0$ if $i<j$. Moreover,

$$
\begin{gathered}
\left(\begin{array}{l}
1 \\
0
\end{array}\right) \Phi_{11}=K_{11}\left(\begin{array}{l}
1 \\
0
\end{array}\right),\left(\begin{array}{l}
0 \\
1
\end{array}\right) \Phi_{22}=K_{22}\left(\begin{array}{l}
0 \\
1
\end{array}\right), \\
\left(\begin{array}{ll|ll}
1 & 0 & 1 & 0 \\
0 & 1 & 0 & 1
\end{array}\right)^{T} \Phi_{33}=\left(K_{33} \oplus K_{44}\right)\left(\begin{array}{ll|ll}
1 & 0 & 1 & 0 \\
0 & 1 & 0 & 1
\end{array}\right)^{T}, \\
\left(\begin{array}{l}
1 \\
1
\end{array}\right) \Phi_{44}=K_{55}\left(\begin{array}{l}
1 \\
1
\end{array}\right), \quad\left(\begin{array}{l}
1 \\
\mu
\end{array}\right) \Phi_{55}=K_{66}\left(\begin{array}{l}
1 \\
\lambda
\end{array}\right) .
\end{gathered}
$$

By the third equality, we obtain $K_{33}=K_{44}$; the first and second equalities yield

$$
K_{11}=K_{22}=\ldots=K_{66}=\left(\begin{array}{ll}
\alpha & 0 \\
0 & \beta
\end{array}\right),
$$

and it follows from the fourth and fifth equalities that $\alpha=\beta$ and $\lambda=\mu$. We have infinitely many nonisomorphic indecomposable spaces $\mathcal{H}_{\lambda}, \lambda \in k$, on $M$. This proves Lemma 2 .

Let $\left(l_{1}, r_{1}\right), \ldots,\left(l_{b} r_{t}\right)$ be all the steps of $M(a, b)$. We set

$$
S(a, b)=\sum_{(i, j)} k e_{i j}^{b a} \quad\left(\operatorname{resp} . \bar{S}(a, b)=\sum_{(i, j)} k e_{i j}^{b a}\right)
$$

where the sum is taken over all $(i, j)$ such that there exists a step $\left(l_{p}, r_{p}\right)>(i, j)\left(\left(l_{p}, r_{p}\right) \geq(i, j)\right.$, respectively). 
Lemma 3. Let $a \neq b$ and $M(a, b)$ have the steps $(1,1),(2,2)$, and $(3,3)$. Then there is no $\psi \in$ $M(a, b)$ such that $M(a, b)=k \psi+S(a, b)$.

Proof. Assume that there exists $\psi \in M(a, b)$ such that $M(a, b)=k \psi+S(a, b)$. By the form of $M(a, b)$ and $\mathcal{A}(b, a) \mathcal{A}(a, b) \subset \mathcal{R}_{\mathcal{A}}(a, a)$, we have $M(b, a) \subset k e_{21}^{a b}+k e_{31}^{a b}+k e_{32}^{a b}$.

Let us examine the space $\mathcal{H}_{\lambda}=\left(k^{3}, h_{\lambda}, a \oplus b\right)$, where $\lambda \in k$ and $h_{\lambda}$ is a linear map from $k^{3}$ into

$$
M(a \oplus b)=k m_{1}^{a} \oplus k m_{2}^{a} \oplus k m_{3}^{a} \oplus k m_{1}^{b} \oplus k m_{2}^{b} \oplus k m_{3}^{b}
$$

with the matrix

$$
\left(\begin{array}{lll|lll}
1 & 0 & 0 & 0 & 0 & 0 \\
0 & 1 & 0 & 0 & 1 & 0 \\
0 & 0 & 1 & 0 & 0 & \lambda
\end{array}\right)^{T}
$$

Let $(\varphi, \xi)$ be an isomorphism $\mathcal{H}_{\lambda} \rightarrow \mathcal{H}_{\mu}$. It follows from the conditions imposed on $M(a, a), M(a, b)$, $M(b, a)$ and $M(b, b)$ that the matrix of $M(\xi)$ has the form

$$
\left(\begin{array}{ccc|ccc}
\alpha_{1} & 0 & 0 & 0 & 0 & 0 \\
\alpha_{2} & \alpha_{1} & 0 & \gamma_{1} & 0 & 0 \\
\alpha_{4} & \alpha_{3} & \alpha_{1} & \gamma_{3} & \gamma_{2} & 0 \\
\hline \delta_{1} & 0 & 0 & \beta_{1} & 0 & 0 \\
\delta_{4} & \delta_{2} & 0 & \beta_{2} & \beta_{1} & 0 \\
\delta_{6} & \delta_{5} & \delta_{3} & \beta_{4} & \beta_{3} & \beta_{1}
\end{array}\right) .
$$

Moreover, $\delta_{1}=\delta \varepsilon_{1}, \delta_{2}=\delta \varepsilon_{2}$, and $\delta_{3}=\delta \varepsilon_{3}$, where $\delta \in k$ and $\varepsilon_{1}, \varepsilon_{2}$, and $\varepsilon_{3}$ are diagonal elements of the lower triangular matrix of $\psi$. By $h_{\mu} \varphi=M(\xi) h_{\lambda}$, we find successively that $\delta=0$, the mapping $\varphi$ has the lower triangular matrix with the diagonal $\left(\alpha_{1}, \alpha_{1}, \alpha_{1}\right), \alpha_{1}=\beta_{1}$, and $\lambda=\mu$.

Hence, $\mathcal{H}_{\lambda} \neq \mathscr{H}_{\mu}$ for $\lambda \neq \mu$ and $M$ is infinitely spaced. We arrive at a contradiction that proves Lemma 3 .

Lemma 4. $S(a, b) \subset M(a, b)$.

Proof. We must show that if $(l, r)$ is a step of $M(a, b)$, then

$$
S_{l r}(a, b)=\sum_{(i, j)<(l, r)} k e_{i j}^{b a} \subset M(a, b)
$$

By Lemma 3, there exists a $\psi \in M(a, b)$ having the step $(l, r)$ but not more than two steps. If $\Psi$ and $M(a, b)$ have the steps $(1,2)$ and $(2,3)$, then, by Lemma $2, e_{31}^{a b} \psi \in M(a, a)$ has the unique step $(3,2)$. Hence,

$$
M(a, a)=k 1_{M(a)} \oplus k e_{21}^{a a} \oplus k e_{31}^{a a} \oplus k e_{32}^{a a} .
$$

In all other cases, it follows from Lemma 1 that $S_{i r}(a, b)$ is contained in the space generated by all $\delta \psi \varepsilon$, where $\varepsilon \in M(a, a)$ and $\delta \in M(b, b)$. This proves Lemma 4 . 
Lemma 4 implies the following assertion:

Lemma 5. Let $a, b \in \mathcal{I A}, a \neq b$, and $M(a, b) \neq \bar{S}(a, b)$. Then only the following three cases can $\operatorname{occur}\left(\lambda_{a b} \neq 0 \neq \mu_{a b}\right)$ :

(a) $M(a, b)$ has two steps $\left(l_{1}, r_{1}\right)$ and $\left(l_{2}, r_{2}\right), l_{1}<l_{2}$, and is equal to

$$
k\left(e_{l_{1} r_{1}}^{b a}+\lambda_{a b} e_{l_{2} r_{2}}^{b a}\right) \oplus S(a, b)
$$

(b) $M(a, b)$ has the steps $(1,1),(2,2)$, and $(3,3)$ and is equal to

$$
k\left(e_{11}^{b a}+\lambda_{a b} e_{22}^{b a}\right) \oplus k e_{33}^{b a} \oplus S(a, b)
$$

or

$$
k\left(e_{11}^{b a}+\lambda_{a b} e_{33}^{b a}\right) \oplus k e_{22}^{b a} \oplus S(a, b)
$$

or

$$
k\left(e_{22}^{b a}+\lambda_{a b} e_{33}^{b a}\right) \oplus k e_{11}^{b a} \oplus S(a, b) ;
$$

(c) $M(a, b)$ has the steps $(1,1),(2,2)$, and $(3,3)$ and is equal to

$$
k\left(e_{11}^{b a}+\lambda_{a b} e_{22}^{b a}\right) \oplus k\left(e_{11}^{b a}+\mu_{a b} e_{33}^{b a}\right) \oplus S(a, b)
$$

Remark 1. In a normed scalarly multiplicative basis, each long double morphism $\varphi \in \mathcal{A}(a, b)$ is a product of double basis morphisms. Indeed, let $\varphi=\tau \psi$, where $\psi \in R_{\mathcal{A}}(a, c)$ and $\tau \in R_{\mathcal{A}}(c, b)$. Then $\psi$ is a unique double morphism of $\mathcal{A}(a, c)$ (otherwise, $\varphi$ is a sum of prime morphisms). Therefore, $\psi$ is a basis morphism. Similarly, $\tau$ is also a basis morphism.

Remark 2. A normed scalarly multiplicative basis is reduced if and only if all long double morphisms are basis morphisms. Indeed, let a long double morphism $\varphi \in \mathcal{A}(a, b)$ be not a basis morphism. Then $\mathcal{A}(a, b)$ has two double basis morphisms and $\varphi$ is their linear combination. But this contradicts the definition of a reduced basis.

Remark 3. Lemma 1 and Lemma 5 imply the statement of Proposition 1 about a normed scalarly multiplicative basis. By Remark 2, to complete the proof of Proposition 1, we must prove that each $\mathcal{A}(a, b)(a, b \in \mathcal{J A})$ does not contain three long double morphisms.

Remark 4. If char $(k) \neq 2$, then every multiplicative basis is reduced. Indeed, otherwise, in view of Remark 2 , there exists a long double morphism $\varphi \in \mathcal{A}(a, b)$ which is not a basis morphism. By virtue of Lemma 5 , $\varphi=\psi-\tau$, where $\psi$ and $\tau$ are basis long double morphisms of $\mathcal{A}(a, b)$. Hence, $M(\varphi)=e_{i i}^{b a}-e_{j j}^{b a}$. Remark 3 implies that $\varphi$ is a product of basis morphisms; hence, $M(\varphi)=e_{i i}^{b a}+e_{j j}^{b a}$ and $\operatorname{char}(k)=2$. 


\section{The Graph of a Scalarly Multiplicative Basis}

In this section, we study some properties of a scalarly multiplicative basis and give the proof of Proposition 1 .

Following [2, Sec. 4.9], we define a poset $\mathscr{P}$ whose elements are the spaces $a_{i}=\left(\mathcal{R}_{\mathfrak{A}}(a, a)\right)^{i-1} M(a)(a \in \mathcal{I A}$, $1 \leq i \leq d(a)$ ) and where $a_{i} \leq b_{j}$ if and only if $\mathcal{A}(b, b) f a_{i}=b_{j}$ for some $f \in \mathcal{A}(a, b)$. The elements $a_{i} \in \mathcal{P}$ are in a one-to-one correspondence with the basis vectors $m_{i}^{a}$ of every scalarly multiplicative basis $\left\{m_{i}^{a}, f_{l}^{b a}\right\}$; moreover, $a_{i}<b_{j}$ if and only if $f_{l}^{b a} m_{i}^{a}=\lambda m_{j}^{a}$ for some $f_{l}^{b a}$ and $0 \neq \lambda \in k$. Decompose the poset $\mathcal{P}$ into the disjoint totally ordered subsets $\left\{a_{1}, \ldots, a_{d(a)}\right\},\left(a_{1}<a_{2}<\ldots<a_{d(a)}, d(a) \leq 3\right)$; each of them is called a double if $d(a)=2$ or a triple if $d(a)=3$.

The following three lemmas were given in [2] without proofs:

Lemma 6 (see [2, Lemma 4.12.1]). The union $\cup\left\{a_{1}, a_{2}, a_{3}\right\}$ of all triples is totally ordered.

Proof. The elements of a triple are totally ordered.

Let $\left\{a_{1}, a_{2}, a_{3}\right\}$ and $\left\{b_{1}, b_{2}, b_{3}\right\}$ be triples and let some $a_{i}$ be not comparable with some $b_{j}$. We construct the indecomposable spaces $\mathcal{H}_{\lambda}=\left(k^{6}, h_{\lambda}, a^{2} \oplus b^{2}\right)$ on $M, \lambda \in k$, such that $\mathcal{H}_{\lambda} \neq \mathcal{H}_{\mu}$ for $\lambda \neq \mu$.

For $i=3$ and $j=1$, the spaces $\mathcal{H}_{\lambda}$ were constructed in the proof of Lemma 2. For arbitrary $i$ and $j, \mathcal{H}_{\lambda}$ is constructed by analogy with the block

$$
\left(\begin{array}{ll|ll}
1 & 0 & 1 & 0 \\
0 & 1 & 0 & 1
\end{array}\right)^{T}
$$

of $h_{\lambda}: k^{6} \rightarrow M\left(a^{2} \oplus b^{2}\right)$ located in the rows of

$$
k m_{i}^{a} \oplus k m_{i}^{a} \oplus k m_{j}^{b} \oplus k m_{j}^{b} \subset M\left(a^{2} \oplus b^{2}\right) .
$$

Let $(\varphi, \xi): \mathcal{H}_{\lambda} \stackrel{\sim}{\rightarrow} \mathcal{H}_{\mu}$ and let $\left(M_{i j}\right)$ be a block matrix of $M(\xi)$. Then $\left(M_{i j}\right)$ is not upper block-triangular, but we can reduce $\left(M_{i j}\right)$ to the upper block-triangular form by means of simultaneous transpositions of vertical and horizontal stripes, since the set $\left\{a_{1}, a_{2}, a_{3}, b_{1}, b_{2}, b_{3}\right\}$ is partially ordered. Hence, $M$ is infinitely spaced. We arrive at a contradiction that proves Lemma 6 .

Lemma 7 (see [2, Lemma 4.9]). There are no the elements $a_{i}, a_{i^{\prime}}, b_{j}$, and $b_{j}$ such that $a_{i} \neq a_{i^{\prime}}$, $b_{j} \neq b_{j^{\prime}}, a_{i}$ is not comparable to $b_{j^{\prime}}$, and $b_{j}$ is not comparable to $a_{i}$. There are no elements $a_{i}, a_{i}, b_{j}, b_{j^{\prime}}$, $c_{l}$, and $c_{l^{\prime}}$ such that $a_{i} \neq a_{i^{\prime}}, b_{j} \neq b_{j^{\prime}}, c_{l} \neq c_{l^{\prime}}, a_{i}$ is not comparable to $b_{j^{\prime}}, b_{j}$ is not comparable to $c_{l^{\prime}}$, and $c_{l}$ is not comparable to $a_{i}$.

Proof. In the first case, we set

$$
\mathcal{H}_{\lambda}=\left(k e_{1} \oplus k e_{2}, h_{\lambda}, a \oplus b\right) \in M^{k}
$$

where $h_{\lambda} e_{1}=m_{i}^{a}+m_{j^{\prime}}^{b}$ and $h_{\lambda} e_{2}=m_{j}^{b}+\lambda m_{i^{\prime}}^{a}$. In the second case, we set

$$
\mathcal{H}_{\lambda}=\left(k e_{1} \oplus k e_{2} \oplus k e_{3}, h_{\lambda}, a \oplus b \oplus c\right),
$$

where $h_{\lambda} e_{1}=m_{i}^{a}+m_{j^{\prime}}^{b}, h_{\lambda} e_{2}=m_{j}^{b}+m_{l^{\prime}}^{c}$, and $h_{\lambda} e_{3}=m_{l}^{c}+\lambda m_{i^{\prime}}^{a}$. Obviously, $\mathcal{H}_{\lambda} \neq \mathcal{H}_{\mu}$ for $\lambda \neq \mu$. 
Lemma 8 (see [2], Lemma 4.12.2]). Each triple contains at least two elements comparable with all elements of all doubles.

Proof. Assume that Lemma 8 is not true for a triple $\left\{a_{1}, a_{2}, a_{3}\right\}$ and doubles $\left\{b_{1}, b_{2}\right\}$ and $\left\{c_{1}, c_{2}\right\}$.

Case I. Assume that $b \neq c$. For definiteness, we assume that $a_{2}$ is not comparable to $b_{1}$ and $a_{3}$ is not comparable to $c_{1}$.

For each representation $\mathcal{H}$

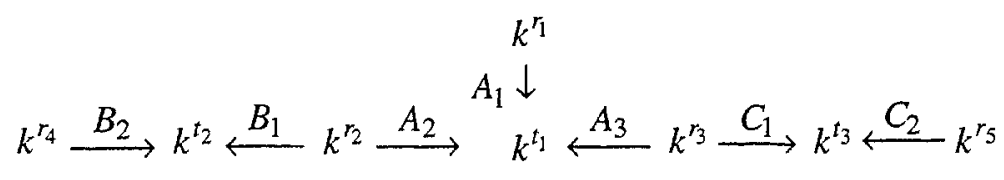

of the quiver $\tilde{E}_{7}$ (see [2, Sec. 6.3]), we construct the space

$$
\overline{\mathcal{H}}=\left(k^{r_{1}}+\ldots+r_{5}, h, a^{t_{1}} \oplus b^{t_{2}} \oplus c^{t_{3}}\right) \in M^{k}
$$

where

$$
h=A_{1} \oplus\left(\begin{array}{l}
A_{2} \\
B_{1}
\end{array}\right) \oplus\left(\begin{array}{l}
A_{3} \\
C_{1}
\end{array}\right) \oplus B_{2} \oplus C_{2}
$$

is a linear mapping of $k^{r_{1}+\ldots+r_{5}}$ into

$$
M\left(a^{t_{1}} \oplus b^{t_{2}} \oplus c^{t_{3}}\right)=\left(k m_{1}^{a}\right)^{t_{1}} \oplus\left[\left(k m_{2}^{a}\right)^{t_{1}} \oplus\left(k m_{1}^{b}\right)^{t_{2}}\right] \oplus\left[\left(k m_{3}^{a}\right)^{t_{1}} \oplus\left(k m_{1}^{c}\right)^{t_{3}}\right] \oplus\left(k m_{2}^{b}\right)^{t_{2}} \oplus\left(k m_{2}^{c}\right)^{t_{3}}
$$

The functor $\mathcal{H} \mapsto \overline{\mathcal{H}}$ on the representations $\mathcal{H}$ with injective $A_{1}, A_{2}, A_{3}, B_{2}$, and $C_{2}$ preserves indecomposability and heteromorphism (i.e., $\mathcal{H} \simeq \mathcal{H}^{\prime}$ if $\left.\overline{\mathcal{H}} \approx \overline{\mathcal{H}}^{\prime}\right)$. Indeed, let $(\varphi, \xi): \overline{\mathcal{H}} \tilde{\rightarrow} \overline{\mathcal{H}}^{\prime}$. The nondegenerate linear maps $\varphi$ and $M(\xi)$ have the block forms $\left(\Phi_{i j}\right), i, j \leq 5$, and $\left(K_{i j}\right), i, j \leq 7$. The equality $h^{\prime} \varphi=$ $M(\xi) h$ implies that $A_{1}^{\prime} \Phi_{11}=K_{11} A_{1}$

$$
\begin{gathered}
\left(\begin{array}{l}
A_{2}^{\prime} \\
B_{1}^{\prime}
\end{array}\right) \Phi_{22}=\left(\begin{array}{ll}
K_{22} & K_{23} \\
K_{32} & K_{33}
\end{array}\right)\left(\begin{array}{l}
A_{2} \\
B_{1}
\end{array}\right), \quad\left(\begin{array}{l}
A_{3}^{\prime} \\
C_{1}^{\prime}
\end{array}\right) \Phi_{33}=\left(\begin{array}{ll}
K_{44} & K_{45} \\
K_{54} & K_{55}
\end{array}\right)\left(\begin{array}{l}
A_{3} \\
C_{1}
\end{array}\right), \\
B_{2}^{\prime} \Phi_{44}=K_{66} B_{2}, \quad C_{2}^{\prime} \Phi_{55}=K_{77} C_{2} .
\end{gathered}
$$

Since $\left\{a_{1}, a_{2}, a_{3}\right\}$ is a triple and $\left\{b_{1}, b_{2}\right\}$ and $\left\{c_{1}, c_{2}\right\}$ are doubles, we have $K_{11}=K_{22}=K_{44}, K_{33}=K_{66}$, and $K_{55}=K_{77}$. Since $a_{2}$ is not comparable to $b_{1}$ and $a_{3}$ is not comparable to $c_{1}$, we have $K_{23}=0, K_{32}=0$, $K_{45}=0$, and $K_{54}=0$. Hence, the diagonal blocks of $\left(\Phi_{i j}\right)$ and $\left(K_{i j}\right)$ determine a morphism $\mathcal{H} \rightarrow \mathcal{H}^{\prime}$.

Let us show that this morphism is an isomorphism, i.e., the diagonal blocks $\Phi_{i i}$ and $K_{i i}$ are invertible. By strengthening the partial order relation in $\left\{a_{1}, a_{2}, a_{3}, b_{1}, b_{2}, c_{1}, c_{2}\right\}$, we obtain a total order relation $\ll$ such that $a_{2} \ll b_{1}$ and $a_{3} \ll c_{1}$ (these pairs are not comparable with respect to $<$ ).

We transpose the horizontal stripes of the matrices of $h$ and $h^{\prime}$ according to the new order. Then we transpose the vertical stripes to get the lower trapezoidal matrices. Correspondingly, we transpose the blocks of ( $\left.\Phi_{i j}\right)$ 
and $\left(K_{i j}\right)$. Then the new matrix $\left(K_{i j}\right)$ has a lower triangular form. The upper nonzero blocks of vertical stripes are the injective maps $A_{1}, A_{2}, A_{3}, B_{2}$, and $C_{2}$ (since $a_{2} \ll b_{1}$ and $a_{3} \ll c_{1}$ ). It follows from $h^{\prime} \varphi=M(\xi) h$ that $\left(\Phi_{i j}\right)$ also has a lower triangular form. Hence, the diagonal blocks $\Phi_{i i}$ and $K_{i i}$ are invertible and $\mathcal{H} \approx \mathcal{H}^{\prime}$.

However, the quiver $\tilde{E}_{7}$ admits an infinite set of nonisomorphic indecomposable representations of the form $\mathscr{H}$ with injective $A_{1}, A_{2}, A_{3}, B_{2}$, and $C_{2}$ (and surjective $B_{1}$ and $C_{1}$, which will be used in case II). These representations are determined by the matrices

$$
\begin{gathered}
\left(A_{1}\left|A_{2}\right| A_{3}\right)=\left(\begin{array}{ll|lll|lll}
1 & \alpha & 1 & 0 & 0 & 0 & 0 & 0 \\
1 & 1 & 0 & 1 & 0 & 0 & 0 & 1 \\
1 & 0 & 0 & 0 & 1 & 0 & 1 & 0 \\
0 & 1 & 0 & 0 & 0 & 1 & 0 & 0
\end{array}\right) \\
\left(B_{1} \mid B_{2}\right)=\left(C_{1} \mid C_{2}\right)=\left(\begin{array}{lll|l}
0 & 1 & 0 & 1 \\
0 & 0 & 1 & 0
\end{array}\right)
\end{gathered}
$$

and they are nonisomorphic for different $\alpha \in k$. This contradicts the assumption that $M$ is finitely spaced.

Case II. Assume that $b=c$. By Lemma 7, if $a_{i}$ is not comparable to $b_{1}$ and $a_{j}$ is not comparable to $b_{2}$, then $i=j$. Let $a_{2}$ and $a_{3}$ be not comparable to $b_{1}$. Then $a_{1}<b_{1}$ and $a_{3}<b_{2}$.

As in case $\mathrm{I}$, for each representation $\mathcal{H}$ of the quiver $\tilde{E}_{7}$ with injective $A_{1}, A_{2}, A_{3}, B_{2}$, and $C_{2}$ and surjective $B_{1}$ and $C_{1}$, we construct the space $\hat{\mathcal{H}}=\left(k^{r_{1}+\ldots+r_{5}}, h, a^{t_{1}} \oplus b^{t_{2}+t_{3}}\right) \in M^{k}$, where

$$
h=A_{1} \oplus\left(\begin{array}{l}
A_{2} \\
B_{1}
\end{array}\right) \oplus\left(\begin{array}{l}
A_{3} \\
C_{1}
\end{array}\right) \oplus B_{2} \oplus C_{2}
$$

is a linear mapping of $k^{r_{1}+\ldots+r_{5}}$ into

$$
M\left(a^{t_{1}} \oplus b^{t_{2}+t_{3}}\right)=\left(k m_{1}^{a}\right)^{t_{1}} \oplus\left[\left(k m_{2}^{a}\right)^{t_{1}} \oplus\left(k m_{1}^{b}\right)^{t_{2}}\right] \oplus\left[\left(k m_{3}^{a}\right)^{t_{1}} \oplus\left(k m_{1}^{b}\right)^{t_{3}}\right] \oplus\left(k m_{2}^{b}\right)^{t_{2}} \oplus\left(k m_{2}^{b}\right)^{t_{3}} .
$$

Let $(\varphi, \xi), \hat{\mathcal{H}} \tilde{\rightarrow} \hat{\mathcal{H}}^{\prime}$. It follows from the order relation for $\left\{a_{1}, a_{2}, a_{3}, b_{1}, b_{2}\right\}$ that all blocks over the diagonal of the block matrix $K=\left(K_{i j}\right)_{i, j=1,2, \ldots, 7}$ of the mapping $M(\xi)$ are zero except the blocks $K_{35}=K_{67}$. Let us prove that they are zero, too.

Indeed, by comparing the blocks with index $(2,3)$ in the equality $h^{\prime} \varphi=M(\xi) h$, we obtain $A_{2}^{\prime} \Phi_{23}=0$ and $\Phi_{23}=0$, since $A_{2}^{\prime}$ is injective. By comparing the blocks with index $(3,3)$, we obtain $B_{1}^{\prime} \Phi_{23}=K_{35} C_{1}$ and $K_{35}=0$, since $C_{1}$ is surjective.

Hence, $K$ is a lower block-triangular matrix. Therefore, $\Phi$ is also a lower block-triangular matrix, whose diagonal blocks $K_{i i}$ and $\Phi_{i i}$ are invertible, $\mathcal{H} \simeq \mathcal{H}^{\prime}$. This proves Lemma 8 .

Now fix a normed scalarly multiplicative basis $\left\{m_{i}^{a}, f_{l}^{b a}\right\}$ and define the oriented graph $\Gamma$, whose set of vertices $\Gamma_{0}$ is a poset $\mathcal{P}$ and there is an arrow $a_{p} \rightarrow b_{q}\left(a_{p}, b_{q} \in \Gamma_{0}\right)$ if and only if $M\left(f_{l}^{b a}\right)=\lambda e_{q p}^{b a}+\mu e_{q^{\prime} p^{\prime}}^{b a}$ for some short double morphism $f_{l}^{b a}$ (in this case, there is an arrow $a_{p^{\prime}} \rightarrow b_{q^{\prime}}$ and we say that the arrows $a_{p} \rightarrow b_{q}$ and $a_{p^{\prime}} \rightarrow b_{q^{\prime}}$ are connected). An arrow $a_{p} \rightarrow b_{q}$ is called a weak arrow if $\mathcal{A}(a, b)$ contains three double morphisms. Each weak arrow is connected with two arrows. The others are called strong arrows, and each of them is connected exactly with one arrow. 
Lemma 9. Let $a_{i}<b_{j}<c_{r}$ and let $a_{i} \rightarrow c_{r}$ be an arrow. Then $a \neq b \neq c \neq a, i=r$, the spaces $\mathcal{A}(a, b), \mathcal{A}(b, c)$, and $\mathcal{A}(a, c)$ contain exactly one, one, and three double morphisms, respectively, and there exists a pair of oriented paths $\left(a_{i} \rightarrow \ldots \rightarrow b_{j} \rightarrow \ldots \rightarrow c_{i}, a_{i^{\prime}} \rightarrow \ldots \rightarrow b_{j^{\prime}} \rightarrow \ldots \rightarrow c_{i^{\prime}}\right)$ consisting of connected strong arrows and a pair of connected weak arrows $\left(a_{i} \rightarrow c_{i}, a_{i^{\prime \prime}} \rightarrow c_{i^{\prime \prime}}\right), i^{\prime} \neq i^{\prime \prime}$. In the case of a reduced scalarly multiplicative basis, there is no other arrow from $\left\{a_{l}\right\}$ to $\left\{c_{l}\right\}$.

Proof. Since $a_{i}<b_{j}<c_{r}$, there are morphisms $g \in \mathcal{A}(a, b)$ and $h \in \mathcal{A}(b, c)$ such that $M(g)=\alpha e_{j i}^{b a}+$ $\beta e_{j^{\prime} i^{\prime}}^{b a}$ and $M(h)=\gamma e_{r j}^{c b}+\delta e_{r^{\prime \prime} j^{\prime \prime}}^{c b}(\alpha, \beta, \gamma, \delta \in k$ and $\alpha \neq 0 \neq \gamma)$. If $h g$ is a prime morphism, then $M(h g)=$ $\alpha \gamma e_{r i}^{c a}$ contradicts the existence of the arrow $a_{i} \rightarrow c_{r}$. Hence, $h g$ is a double morphism, $\beta \neq 0 \neq \delta, j^{\prime}=j^{\prime \prime}$, and $g$ and $h$ are unique double morphisms of $\mathcal{A}(a, b)$ and $\mathcal{A}(b, c)$, respectively. The space $\mathcal{A}(a, c)$ contains the double morphism $h g$ and the short double morphism corresponding to the arrow $a_{i} \rightarrow c_{r}$, whence $M(a, c)$ has the form given in case (c) of Lemma 5.

If the basis is reduced, then, by Remark 2 in Sec. 1, the double morphism $h g$ is a basis morphism and there is only one pair of connected arrows from $\left\{a_{l}\right\}$ to $\left\{c_{l}\right\}$. This proves Lemma 9 .

Proof of Proposition 1. In view of Remark 3 in Sec. 1, we must prove that each space $\mathcal{A}(a, c)(a, c \in \mathcal{I A})$ does not contain three long double morphisms. By contradiction, let $f_{1}, f_{2}, f_{3} \in \mathcal{A}(a, c)$ be three long double morphisms and let $f_{r}=h_{r} g_{r}$, where $g_{r}$ is a short double morphism and $r=1,2,3$. The morphisms $g_{1}, g_{2}$, and $g_{3}$ correspond to the pairs of connected arrows $\left(a_{1} \rightarrow x_{i}, a_{2} \rightarrow x_{i^{\prime}}\right),\left(a_{1} \rightarrow y_{j}, a_{3} \rightarrow y_{j^{\prime}}\right)$, and $\left(a_{2} \rightarrow z_{l^{\prime}}\right.$, $\left.a_{3} \rightarrow z_{l^{\prime}}\right)$.

Assume that $x_{i}<y_{j}$. By putting $\left(a_{i}, b_{j}, c_{r}\right)=\left(a_{1}, x_{i}, y_{j}\right)$ in Lemma 9, we establish that $\mathcal{A}(a, y)$ contains three double morphisms. By putting $\left(a_{i}, b_{j}, c_{r}\right)=\left(a_{1}, y_{j}, c_{1}\right)$ in Lemma 9 , we obtain that $\mathcal{A}(a, y)$ contains exactly one double morphism.

Hence, $x_{i}$ is not comparable to $y_{j}$. Similarly, $x_{i^{\prime}}$ is not comparable to $z_{l}$ and $y_{j^{\prime}}$, is not comparable to $z_{l^{\prime}}$. This contradicts Lemma 7 and proves Proposition 1.

Assume that the graph $\Gamma$ is obtained from a reduced scalarly multiplicative basis.

Lemma 10. If two arrows start from (stop at) the same vertex, then the arrows connected with them start from (stop at) different vertices.

Proof. By contradiction, let $b_{j} \leftarrow a_{i} \rightarrow c_{r}$ and $b_{j^{\prime}} \leftarrow a_{i^{\prime}} \rightarrow c_{r^{\prime}}$ be connected arrows. If $b_{j}<c_{r}$, then $a_{i}<b_{j}<c_{r}$ and, by Lemma 9 , the arrows connected with $a_{i} \rightarrow b_{j}$ and $a_{i} \rightarrow c_{r}$ must start from different vertices, but they start from $a_{i}$. By analogy, $b_{j^{\prime}}$ is not comparable to $c_{r^{\prime}}$. This contradicts Lemma 7.

Lemma 11. There are no two arrows starting from (stopping at) the same vertex of a double. There are no three arrows starting from (stopping at) the same vertex of a triple.

The proof follows from Lemma 10.

Lemma 12. There are at most two different pairs of connected arrows starting from (stopping at) the same triple.

Proof. By contradiction, assume that three pairs of connected arrows from a triple $\left\{a_{1}, a_{2}, a_{3}\right\}$ to $\left\{b_{i}\right\}$, $\left\{c_{i}\right\},\left\{d_{i}\right\}$ exist. Since there are at most two pairs of connected arrows from a triple to a triple, we do not have three coinciding objects among $a, b, c$, and $d$. Hence, the following five cases are possible (up to the permutation of $b, c, d)$ : 
(I) $a=b \neq c \neq d, a \neq d$;

(II) $a=b \neq c=d$;

(III) $a \neq b=d \neq c, a \neq c$;

(IV) $a, b, c, d$ are distinct and there are two arrows $a_{i} \rightarrow b_{j}$ and $a_{i} \rightarrow c_{r}, b_{j}<c_{r}$;

(V) $a, b, c, d$ are distinct and, for each pair of arrows $a_{i} \rightarrow x, a_{i} \rightarrow y$, the vertices $x$ and $y$ are incomparable.

By Lemmas 9-11, we have the following subgraphs of $\Gamma$ in cases (I), (III), and (IV):

(I)

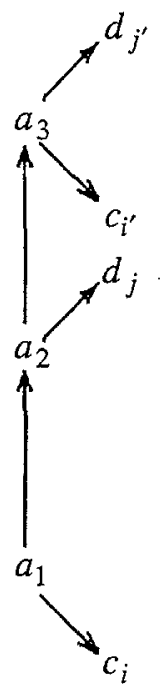

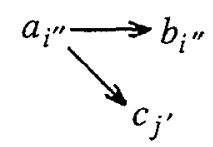

(III)
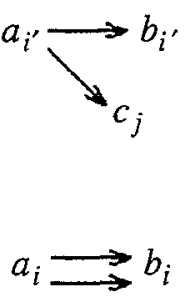

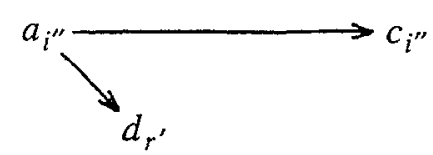

(IV)
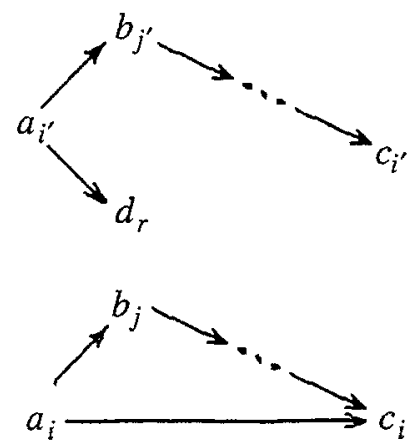

Consider these cases.

(I) If $c_{i}<a_{2}$ or $d_{j}<a_{3}$, then, by Lemma $9, \mathcal{A}(a, a)$ contains three double morphisms, which is a contradiction. If $a_{2}<c_{i}$ or $a_{3}<d_{j}$, then, in view of Lemma 9, there is an arrow $a_{2} \rightarrow c_{i}$ or $a_{3} \rightarrow d_{j}$, in contradiction with Lemma 11. Hence, $a_{2}$ is incomparable with $c_{i}$ and $a_{3}$ is incomparable with $d_{j}$, which is impossible by Lemma 8.

(II) This case is similar to the previous one.

(III) By Lemma 9, the inequality $b_{i},<c_{j}$ is impossible because $\mathcal{A}(a, b)$ contains three double morphisms. Lemma 9 also implies that the inequality $b_{i^{\prime}}>c_{j}$ is impossible because there are four arrows from $\left\{a_{l}\right\}$ to $\left\{b_{l}\right\}$. Hence, $b_{i^{\prime}}$ is incomparable with $c_{j}$. By analogy, $b_{i^{\prime \prime}}$ is not comparable to $c_{j^{\prime}}$ in contradiction with Lemma 7 .

(IV) The inequalities $c_{i^{\prime}}<d_{r}$ and $c_{i^{\prime \prime}}<d_{r^{\prime}}$ are impossible by virtue of Lemma 9 because $\mathcal{A}(a, c)$ contains three double morphisms. If $d_{r}<c_{i^{\prime}}$ or $d_{r^{\prime}}<c_{i^{\prime \prime}}$, then the double morphism $\lambda e_{i^{\prime} i^{\prime}}^{c a}+\mu e_{i^{\prime \prime} i^{\prime \prime}}^{c a}(\lambda \neq 0 \neq \mu)$ is a product of double morphisms in $\mathcal{A}(a, d)$ and $\mathcal{A}(d, c)$. Hence, $\mathcal{A}(a, c)$ contains two long double morphisms in 
contradiction with the arrows $a_{i} \rightarrow c_{i}$ and $a_{i^{\prime \prime}} \rightarrow c_{i}$ ". This implies that $c_{i^{\prime}}$ is not comparable to $d_{r}$ and $c_{i^{\prime \prime}}$ is not comparable to $d_{r^{\prime}}$, which contradicts Lemma 7.

(V) This case is impossible by virtue of Lemma 7.

The proof of Lemma 12 is thus completed.

\section{The Construction of a Multiplicative Basis}

In this section, we prove the following proposition.

Proposition 2. From every reduced scalarly multiplicative basis, we can obtain a reduced multiplicative basis by multiplying the basis vectors by nonzero elements of $k$.

Let $\Gamma$ be a graph of a reduced scalarly multiplicative basis $\left\{m_{i}^{a}, f_{l}^{b a}\right\}$ and let $\Gamma_{1}$ be a set of its arrows. An integral function $z: \Gamma_{1} \rightarrow \mathbb{Z}$ is called a weight function and its value at an arrow is called the weight of the arrow if

(a) $z\left(\alpha_{1}\right)=-z\left(\alpha_{2}\right)$ for each pair of connected arrows $\alpha_{1}, \alpha_{2}$;

(b) the sum of the weights of all arrows stopping at a vertex $v \in \Gamma_{0}$ is equal to the sum of the weights of all arrows starting from $v$ (this sum is called a weight of $v$ and is denoted by $z(v)$ ).

Lemma 13. A nonzero weight function does not exist.

Proof. By contradiction, let $z: \Gamma_{1} \rightarrow \mathbb{Z}$ be a nonzero weight function. An arrow $\alpha$ is called nondegenerate if $z(\alpha) \neq 0$.

Let $v_{1}<\ldots<v_{m}$ be the set of all vertices of the triples of $\Gamma$. For each vertex $v_{i}$, we denote by $v_{i}, v_{i}$, the two vertices such that $\left\{v_{i}, v_{i^{\prime}}, v_{i^{\prime \prime}}\right\}$ is a triple.

By an elementary path of length $s$, we mean a sequence of arrows of the form

$$
v_{p} \stackrel{\lambda_{1}}{\longrightarrow} u_{1} \stackrel{\lambda_{2}}{\longrightarrow} u_{2} \longrightarrow \ldots \longrightarrow u_{s-1} \stackrel{\lambda_{s}}{\longrightarrow} v_{q}
$$

where $u_{1} \ldots, u_{s-1}$ are vertices of doubles (they may be absent, i.e., a path may consist of exactly one arrow) and $z\left(\lambda_{1}\right) \neq 0$. Then, by Lemma 11 and item (b) of the definition of a weight function, $z\left(\lambda_{1}\right)=z\left(\lambda_{2}\right)=\ldots=z\left(\lambda_{s}\right)$; this nonzero integer is called the weight of path (2). We say that the elementary path (2) avoids a vertex $v_{i}$ if $p<i<q$. We now establish some properties of elementary paths.

A. The intersection of two elementary paths does not contain any vertex of a double.

B. Each nondegenerate arrow is contained in an elementary path.

C. If a vertex $v_{i}$ is avoided by an elementary path (2) having length at least two, then $v_{i}$ is incomparable with some vertex $u_{l}$ in this path. Otherwise, $v_{p}<u_{1}<\ldots<u_{s-1}<v_{q}$ implies one of the following conditions: $v_{p}<v_{i}<u_{1}$ or $u_{j}<v_{i}<u_{j+1}$ for some $j$ or $u_{s-1}<v_{i}<v_{q}$. This contradicts Lemma 9 because the vertices $u_{1}, \ldots, u_{s-1}$ are contained in doubles. 
D. If a vertex of a triple is avoided by an elementary path of length at least two, then all other vertices of this triple cannot be avoided by any elementary path of length $\geq 2$. This follows from property $\mathrm{C}$ and Lemma 8 .

$\mathrm{E}$. The sum of the weights of all elementary paths avoiding a vertex $v_{i}$ is equal to $-z\left(v_{i}\right)$. Indeed, this is obvious for $v_{1}$ because, by property $\mathrm{B}$, only arrows having weight zero can stop at $v_{1}$. If property $\mathrm{E}$ is true for $v_{i}$, then the sum of the weights of all elementary paths avoiding $v_{i}$ or starting from $v_{i}$ is equal to zero. But the set of these paths coincides with the set of all elementary paths avoiding $v_{i+1}$ or stopping at $v_{i+1}$. Hence, property E is true for $v_{i+1}$.

F. Let a triple $\left\{b_{1}, b_{2}, b_{3}\right\}$ satisfy the following conditions:

(i) there is no nondegenerate arrow starting from $a<b_{1}$;

(ii) there is a pair of connected degenerate strong arrows starting from $\left(b_{1}, b_{2}\right)$ or $\left(b_{1}, b_{3}\right)$;

(iii) there is a pair of connected nondegenerate weak arrows starting from $\left(b_{2}, b_{3}\right)$.

Then there exists a triple $\left\{a_{1}, a_{2}, a_{3}\right\}$ satisfying the same conditions and relation $a_{1}<b_{1}$. Indeed, let, for definiteness, the pair of connected degenerate strong arrows start from $\left(b_{1}, b_{2}\right)$. It follows from $z\left(b_{1}\right)=0$, $z\left(b_{2}\right)=-z\left(b_{3}\right) \neq 0$, and properties $\mathrm{D}$ and $\mathrm{E}$ that $b_{2}$ or $b_{3}$ is avoided by a nondegenerate arrow. Let $b_{3}$ be avoided by a nondegenerate arrow $a_{i} \rightarrow c_{j}$. Then $a_{i}<b_{3}<c_{j}$. By Lemma 9 , there exists a path $a_{i} \rightarrow \ldots \rightarrow$ $b_{3} \rightarrow \ldots \rightarrow c_{j}$ consisting of strong arrows. However, by Lemma 12, there is only a weak arrow starting from $b_{3}$. Hence, $b_{2}$ is avoided by some nondegenerate arrow $a_{i} \rightarrow c_{j}$. By Lemma 9, it is a weak arrow, $i=j$, and there is a path $a_{i} \rightarrow \ldots \rightarrow b_{2} \rightarrow \ldots \rightarrow c_{i}$ consisting of strong arrows. However, there exists only one strong arrow starting from $b_{2}$ and it is connected with an arrow starting from $b_{1}$. Hence, the arrows connected with $a_{i} \rightarrow \ldots \rightarrow b_{2} \rightarrow \ldots \rightarrow c_{i}$ compose the path $a_{i^{\prime}} \rightarrow \ldots \rightarrow b_{1} \rightarrow \ldots \rightarrow c_{i^{\prime}}$. The triple $\left\{a_{1}, a_{2}, a_{3}\right\}$ satisfies our requirements.

Let $c_{l}$ be a vertex such that there is a nondegenerate arrow starting from $c_{l}$ and there is no nondegenerate arrow starting from $b<c_{l}$. Then there is no nondegenerate arrow stopping at $c_{l}$. Hence, $z\left(c_{l}\right)=0$ and there are two arrows starting from $c_{l}$ and having weights $n$ and $-n$; moreover, $l=1$ and the arrows connected with them start from $c_{2}$ and $c_{3}$. Since $z\left(c_{2}\right)=-z\left(c_{3}\right)= \pm n \neq 0$, the vertices $c_{2}$ and $c_{3}$ are avoided by elementary paths, and one of them is a nondegenerate arrow. For definiteness, assume that $c_{2}$ is avoided by a nondegenerate arrow $b_{i} \rightarrow d_{j}$. By Lemma 9, $i=j$ and there exists a path $b_{i} \rightarrow \ldots \rightarrow c_{2} \rightarrow \ldots \rightarrow d_{i}$. Since there exists exactly one arrow starting from $c_{2}$ and this arrow is connected with an arrow starting from $c_{1}$, we conclude that the arrows connected with $b_{i} \rightarrow \ldots \rightarrow c_{2} \rightarrow \ldots \rightarrow d_{i}$ compose the path $b_{i^{\prime}} \rightarrow \ldots \rightarrow c_{1} \rightarrow \ldots \rightarrow d_{i^{\prime}}$. Since $b_{i^{\prime}}<c_{1}$, there is no nondegenerate arrow starting from $b_{i^{\prime}}$. Hence, the arrow $b_{i} \rightarrow d_{i}$ is connected with the arrow $b_{i \prime \prime} \rightarrow d_{i \prime \prime}$, where $i^{\prime} \neq i^{\prime \prime}$ and $i^{\prime}=1$. By applying property $\mathrm{F}$ to the triple $\left\{b_{1}, b_{2}, b_{3}\right\}$, we obtain a triple $\left\{a_{1}, a_{2}, a_{3}\right\}$. By applying property $\mathrm{F}$ to the triple $\left\{a_{1}, a_{2}, a_{3}\right\}$, we obtain another triple and so on. This contradicts the finiteness of the graph $\Gamma$ and proves Lemma 13.

Proof of Proposition 2. Number all the vertices and all the arrows of the graph $\Gamma$ :

$$
\Gamma_{0}=\left\{a_{1}, a_{2}, \ldots, a_{r}\right\}, \quad \Gamma_{1}=\left\{f_{11}, f_{12}, \ldots, f_{s 1}, f_{s 2}\right\},
$$

where $f_{j 1}: a_{p(j 1)} \rightarrow a_{q(j 1)}$ and $f_{j 2}: a_{p(j 2)} \rightarrow a_{q(j 2)}$ are two connected arrows and $a_{p(j 1)}<a_{p(j 2)}$. Let the basis 
vector $m_{i}$ correspond to the vertex $a_{i}$ and let the double morphism $f_{i}$ correspond to the pair $\left(f_{j 1}, f_{j 2}\right)$. Then $f_{j} m_{p(j 1)}=m_{q(j 1)}$ and $f_{j} m_{p(j 2)}=\lambda_{j} m_{q(j 2)}$, where $\lambda_{j}$ is a parameter of a double morphism $f_{j}$.

By changes of the basis vectors

$$
m_{i}=x_{i} m_{i}^{\prime}, \quad 0 \neq x_{i} \in k
$$

we obtain a new set of double morphisms $f_{j}^{\prime}=x_{p(j 1)} x_{q(j 1)}^{-1} f_{j}, 1 \leq j \leq s$, with the parameters

$$
\lambda_{j}^{\prime}=\lambda_{j} x_{p(j 1)} x_{q(j 1)}^{-1} x_{p(j 2)}^{-1} x_{q(j 2)}
$$

Change (3) gives a multiplicative basis if $\lambda_{1}^{\prime}=\lambda_{2}^{\prime}=\ldots=\lambda_{s}^{\prime}=1$, i.e., if $x_{1}, x_{2}, \ldots, x_{r}$ satisfy the system of equations

$$
\lambda_{j} x_{p(j 1)} x_{p(j 2)}^{-1}=x_{q(j 1)} x_{q(j 2)}^{-1}, \quad 1 \leq j \leq s
$$

We solve the system by elimination, namely, we solve the first equation for some $x_{i}$ and substitute the result in the other equations. This amounts to the multiplication of each of them by a rational power of the first equation. Further, we solve the second equation of the obtained system for some $x_{j}$ and substitute the result in the other equations, etc. There are two possibilities.

I. After the $s$ th step, we obtain the solution $\left(x_{1}, \ldots, x_{t}\right) \in(k \backslash\{0\})^{t}$ of (4).

II. After the $(t-1)$ th step $(1<t \leq s)$, we obtain a system, the $t$ th equation of which does not contain unknowns. In this case, the $t$ th equation of (4), up to scalar multiples $\lambda_{t}$, is the product of rational powers of the 1 st, $\ldots,(t-1)$ th equations. This means that there exist integers $z_{1}, \ldots, z_{t}$ such that $z_{t} \neq 0$ and the equality

$$
\left(x_{p(11)} x_{p(12)}^{-1}\right)^{z_{1}} \ldots\left(x_{p(t 1)} x_{p(t 2)}^{-1}\right)^{z_{t}}=\left(x_{q(11)} x_{q(12)}^{-1}\right)^{z_{1}} \ldots\left(x_{q(t 1)} x_{q(t 2)}^{-1}\right)^{z_{t}}
$$

is the identity, i.e., each $x_{i}$ has the same exponents at the two sides of (5).

Define an integer function $z: \Gamma_{1} \rightarrow \mathbb{Z}$ by $z\left(f_{j 1}\right)=-z\left(f_{j 2}\right)=z_{j}$ for $j \leq t$ and $z\left(f_{j 1}\right)=z\left(f_{j 2}\right)=0$ for $j>t$. Since $x_{i}$ corresponds to the vertex $a_{i}$ of $\Gamma$, we have by (5) that this function is a nonzero weight function, which contradicts Lemma 13. Hence, case II is impossible. This completes the proof of Proposition 2.

\section{REFERENCES}

1. R. Bautista, P. Gabriel, A. V. Roiter, and L. Salmeron, "Representations of finite algebras and multiplicative bases," Invent. Math., 81, 217-285 (1985).

2. P. Gabriel and A. V. Roiter, "Representations of finite-dimensional algebras," in: Encyclopaedia of Mathematical Sciences, Vol. 73, Algebra 8, Springer (1992).

3. P. Gabriel, L. A. Nazarova, A. V. Roiter, V.V. Sergeichuk, and D. Vossieck, "Tame and wild subspace problems," Ukr. Mat. Zh., 45, No. 3, 313-352 (1993). 